\title{
Impaired Autonomic Vascular Control: A Non Linear Dynamic Analysis
}

\author{
G Merati $^{1,3}$, M Di Rienzo ${ }^{2}$, A Veicsteinas ${ }^{1}$, P Castiglioni $^{2}$ \\ ${ }^{1}$ Institute of Physical Exercise, Health and Sports (IEFSAS), University of Milan, Milan, Italy \\ ${ }^{2}$ Centre of Bioengineering and ${ }^{3}$ Centre of Sports Medicine, Don Gnocchi Foundation, Milan, Italy
}

\begin{abstract}
The specific role of autonomic vascular control in determining non-linear behaviour of RR intervals is not clear. In $10 T_{12}-L_{4}$ paraplegic subjects (light vascular autonomic impairment), $12 T_{5}-T_{11}$ paraplegic subjects (moderate vascular autonomic impairment), and 24 controls $R R$ time series were recorded (10 $\mathrm{min}): 1)$ in resting supine position; 2) sitting on the wheelchair and 3) during a light (5W) arm exercise. Fractal Dimension (FD), Approximate Entropy (ApEn) and the $\alpha$ scaling exponent ( $\alpha-D F A$, Detrended Fluctuation Analysis) were calculated. The lesional level significantly affected ApEn $(p=0.004), \quad F D \quad(p=0.007) \quad$ and $\alpha-D F A \quad(p=0.002)$. Autonomic activation did not influence $F D$ and $\alpha-D F A$, but significantly affected ApEn $(p=0.035)$. It seems that $F D$ and $\alpha$-DFA are influenced by the changes in nonlinear RR dynamics due to vascular autonomic impairment but not to autonomic activation, whereas ApEn may be equally responsive to either condition.
\end{abstract}

\section{Introduction}

Pathologies affecting both cardiac and vascular autonomic innervation (atherosclerosis, diabetic neuropathy, etc.) have been shown to influence nonlinear behaviour of heart rate (HR)[1]. However, the specific role of autonomic vascular control in determining non-linear behaviour of RR intervals is not fully understood, probably due to the lack of appropriate physiopathological models, in which the role of heart innervation can be distinguished by that of vascular innervation.

In paraplegic patients the transverse section of spinal cord causes a variable impairment of the autonomic tone, which is dependent on the lesional level[2]. If the spinal cord injury (SCI) is below $\mathrm{T}_{5}$, the autonomic innervation of the heart is preserved, whereas the vascular innervations of the splanchnic area and lower limbs are compromised. Theoretically, below $\mathrm{T}_{5}$ level the higher the lesion, the more severe the autonomic impairment.
We previously demonstrated that the spectral component which mostly reflects vascular tone, i.e. the $0.1 \mathrm{~Hz}$ rhythm, is variably affected in SCI patient, depending on the lesional level below $\mathrm{T}_{5}[3,4]$.

To date, the role of the lesional level in determining the autonomic impairment in paraplegic and tetraplegic subjects have been mainly investigated by spectral analysis of heart rate variability[5,6], whereas non linear component of heart rate variability has been poorly studied.

Aim of this study was to assess the changes in non linear features of HR caused by different degrees of autonomic activation (1.light autonomic activation: transition from the supine to the sitting position, and 2.moderate autonomic stimulus: steady-state exercise) in healthy subjects and in patients with different vascular autonomic impairment (but preserved heart innervation) due to different lesion levels.

\section{Materials and methods}

\subsection{Subjects}

Twelve SCI subjects with lesion level at $\mathrm{T}_{12}-\mathrm{L}_{4}$ (light vascular autonomic impairment), 10 SCI patients with lesional level at $\mathrm{T}_{5}-\mathrm{T}_{11}$ (moderate vascular autonomic impairment), and 24 control subjects were enrolled. All SCI were traumatic and stabilised (lesion duration $>15$ years), and both patients and controls were non smokers and did not present other pathologies which could further affect the autonomic tone (diabetes, hypertension, etc.).

All the subjects signed their informed consent to participate to the study, and the experimental protocol was approved by the local Ethic Committee (Don C. Gnocchi Foundation).

The anthropometric features of the participants are shown in table 1. 
Table 1. Anthropometric features of the enrolled subjects $(\mathrm{m} \pm \mathrm{SD}) . S C I=$ spinal cord injury; $C N T=$ control; $* \mathrm{p}<0.05$ (1-way ANOVA).

\begin{tabular}{lccc}
\hline & SCI & SCI & CNT \\
& $\left(\mathrm{T}_{12}-\mathrm{L}_{4}\right)$ & $\left(\mathrm{T}_{5}-\mathrm{T}_{11}\right)$ & \\
\hline $\mathrm{N}$ & 10 & 12 & 24 \\
Gender, M/F & $9 / 1$ & $12 / 0$ & $19 / 5$ \\
Age, $\mathrm{yrs}$ & $44 \pm 9 *$ & $34 \pm 6$ & $31 \pm 8$ \\
BMI, $\mathrm{kg} / \mathrm{m}^{2}$ & $25.7 \pm 4.1$ & $22.3 \pm 4.3$ & $23.7 \pm 3.1$ \\
Lesion duration, y & $17 \pm 10$ & $19 \pm 9$ & - \\
\hline
\end{tabular}

\subsection{Experimental procedure}

Before the beginning of the experiments, all subjects underwent a medical and physical examination, including the measurement of resting arterial blood pressure. None of the subjects showed signs of cardiorespiratory disease.

The ECG was monitored and recorded during three different experimental conditions: 1) supine, at rest; 2) sitting on the wheelchair (light autonomic activation); 3 ) during a light $(5 \mathrm{~W})$ steady-state exercise by an arm ergometer (Monark 881, Sweden), (moderate autonomic activation).

The ECG signal was acquired at $200 \mathrm{~Hz}$ by a digital acquisition board (DAQ Board, National Instrument, USA) and stored on a computer for off-line analysis. Each recording lasted at least 10 minutes. RR interval series were derived on a beat-by-beat basis from the recorded signals. Original records were manually edited to remove artefacts and ectopic beats.

\subsection{Calculations}

The following parameters were assessed on RR series: Fractal Dimension (FD, which quantifies the space-filling propensity of a time series), Approximate Entropy (ApEn: $\mathrm{n}=500 ; \mathrm{m}=2 ; \mathrm{r}=0.2 * \mathrm{SD}$ of time series, which quantifies the regularity of time series) and the $\alpha$ scaling exponent from a Detrended Fluctuation Analysis $(\alpha-$ DFA, which quantifies the fractal correlation properties of time series).

FD, which measures the scale independence of a system over time, was determined by the method of the "dispersional analysis", a technique which is particularly insensitive to noise and can be applied to short data set of biological signals[7]. Briefly, the SD of the original signal was firstly calculated. Two consecutive data points were then replaced by their mean, halving the total number of data points. The SD of this new data set was then recalculated. This process was repeated iteratively until the number of data point was $\leq 4$. The log of the SD was finally plotted against the $\log$ of $m$, where $m$ is the number of points grouped. If the linearity of the plot was confirmed by a linear regression analysis the FD was calculated as:

$$
\mathrm{FD}=1-s
$$

where $s$ is the slope of the regression line[7].

According to the original method of Pincus et al.[8] ApEn was derived by the correlation integral $\mathrm{C}(r)$, i.e. the number of points in the signal closer than distance $r$ to the $i$ th point when embedded in an $m$-dimensional space. ApEn was calculated as:

where

$$
\operatorname{ApEn}(m, r)=\Phi^{m}(r)-\Phi^{m+1}(r)
$$

$$
\Phi^{m}(r)=\sum_{\mathrm{i}=1}^{\mathrm{N}-m+1} \frac{\ln \mathrm{C}^{\mathrm{m}}(\mathrm{r})}{\mathrm{N}-m+1}
$$

According to Pincus, in RR series reasonable values of ApEn can be obtained for $m=2$ and $r=0,2$ SD of the time series. We also used these parameters in our calculations.

DFA is a method suitable for the analysis of non stationary time series, which quantifies the fractal-like scaling properties of RR data[9]. The RMS fluctuation of integrated and detrended time series was measured at each observation window and plotted against the size of the observation window on a log-log-scale. The slope of this line gave the scaling exponent $d$, which was related to the " $1 / \mathrm{f}$ " spectral slope $\alpha$ :

$$
\alpha=2 d-1
$$

\subsection{Statistics}

Data were expressed as $\mathrm{m} \pm \mathrm{SD}$. Group comparisons were performed by a two-way ANOVA test, by which the effects "lesional level" and "autonomic activation" on FD, ApEn and $\alpha$-DFA were evaluated. The level of statistical significance was set at $\mathrm{p}<0.05$.

\section{Results}

Table 2 shows the HR values during the different experimental conditions and the arterial blood pressure measured at rest. No significant differences were found between groups for all these parameters, except for SBP in $\mathrm{T}_{12}-\mathrm{L}_{4} \mathrm{SCI}$ and exercise HR in $\mathrm{T}_{5}-\mathrm{T}_{11}$ SCI subjects.

Table 2. Heart rate (HR) in the different experimental conditions and basal blood pressure $(\mathrm{m} \pm \mathrm{SD}) . S C I=$ spinal cord injury; $C N T=$ control; ${ }^{*} \mathrm{p}<0.05$ (1-way ANOVA).

\begin{tabular}{lccc}
\hline & SCI & SCI & CNT \\
& $\left(\mathrm{T}_{12}-\mathrm{L}_{4}\right)$ & $\left(\mathrm{T}_{5}-\mathrm{T}_{11}\right)$ & \\
\hline HR supine, ms & $903 \pm 141$ & $838 \pm 161$ & $950 \pm 150$ \\
HR sitting, ms & $895 \pm 124$ & $781 \pm 175$ & $822 \pm 133$ \\
HR exercise, ms & $781 \pm 99$ & $653 \pm 134 *$ & $740 \pm 116$ \\
SBP/DBP, mmHg & $139 * / 10$ & $124 / 21$ & $125 / 15$ \\
\hline
\end{tabular}


FD was significantly affected by the lesion level ( $\mathrm{p}=0.007$ ), but was not responsive to autonomic activation (Figure 1).

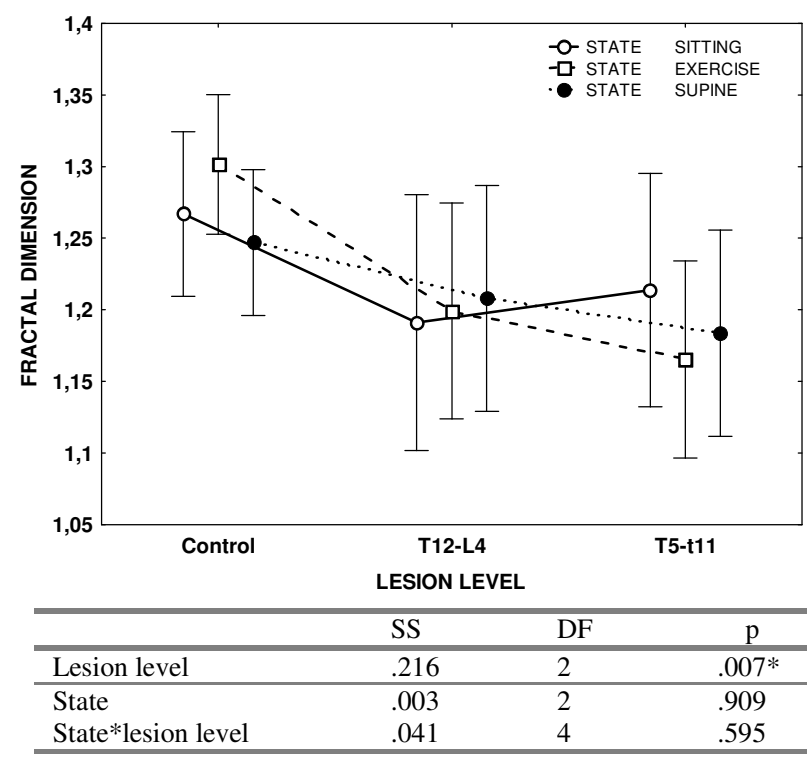

Figure 1: Fractal Dimension (FD)

A similar response was observed for $\alpha$-DFA, which was significantly affected $(\mathrm{p}=0.002)$ by the lesion level but not by autonomic activation (Figure 2 ).

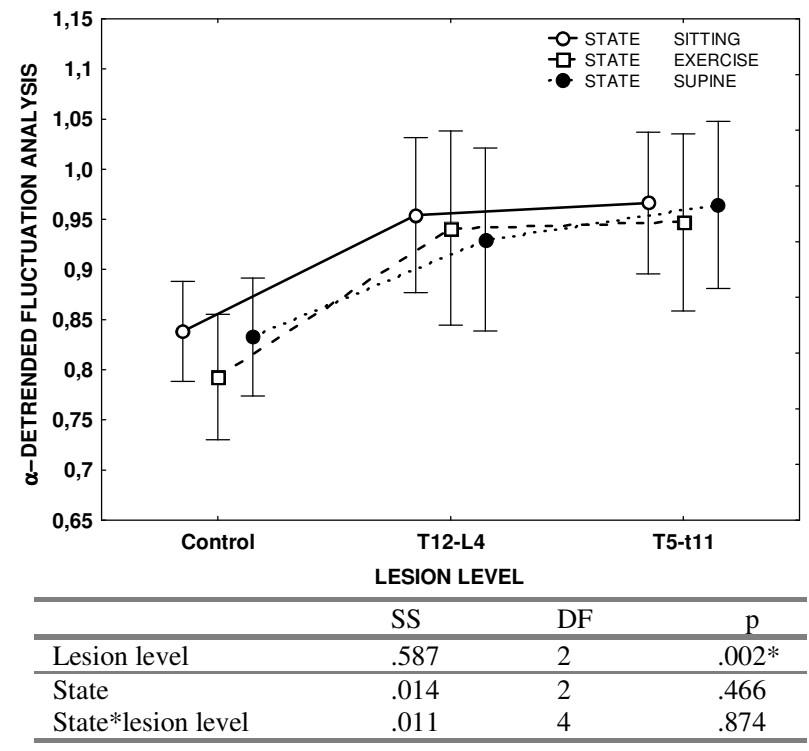

Figure 2: $\alpha$-scaling exponent of DFA ( $\alpha$-DFA)

Conversely, ApEn was significantly affected by both the lesion level $(\mathrm{p}=0.004)$ and by the autonomic activation $(\mathrm{p}=0.035)$. Nevertheless, the interaction of the two effects was not significant (Figure 3).

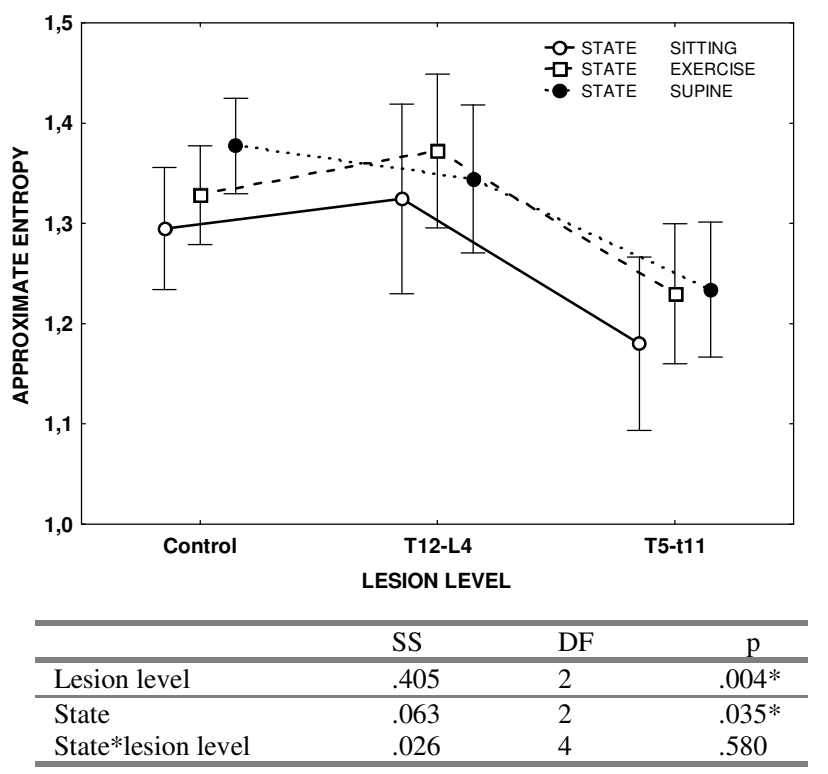

Figure 3: Approximate Entropy (ApEn)

\section{Discussion}

Our results suggest that both FD and $\alpha$-DFA are sensitive to the changes in vascular autonomic tone due to a spinal cord lesion, but fail to reflect the changes in non-linear RR dynamics caused by vascular autonomic activation.

On the other hand, ApEn appears to be the only nonlinear parameter equally responsive to both conditions. It seems therefore that FD and $\alpha$-DFA can be indifferently used only in those comparisons where the impairment of the autonomic tone due to any pathology can be detected in resting, steady-state conditions. However, when a provocative study has to be performed (i.e. in the presence of stimuli that activate the autonomic nervous system, like exercise or postural changes), only ApEn seems to elicit an acceptable resolution of the autonomic changes investigated in the RR series. Moreover, ApEn has been already demonstrated to be highly sensitive to the changes in non-linear RR dynamics occurring during physical exercise [10].

However, we cannot exclude that different physiological correlates of these non linear indexes might play a role. Further studies are therefore needed to elucidate the various factors underlying the vascular adaptation to autonomic activation and their possible effects on the non-linear features of heart rate variability. 


\section{References}

[1] Mestivier D, Chau NP, Chanudet X, Bauduceau B, Larroque $\mathrm{P}$. Relationship between diabetic autonomic dysfunction and heart rate variability assessed by recurrence plot. Am J Physiol 1997;272:H1094-9.

[2] Mathias CJ, Frankel HL. Cardiovascular control in spinal man. Ann Rev Physiol 1988;50:577-592

[3] Di Rienzo M, Castiglioni P, Parati G, Mancia G, Veicsteinas A, Pedotti A, Merati G. Cardiovascular variability and baroreflex sensitivity in individuals with spinal cord injury below T4. Proceedings of the 11th European Meeting on Hypertension, Milan, 2001

[4] Merati G, Tordi R, Di Rienzo M, Veicsteinas A, Castiglioni P. Heart rate and blood pressure variability at rest and during exercise in patients with traumatic spinal cord injury. Proceedings of the Congress of the European College of Sports Medicine, Rome, 1999

[5] Inoue K, Ogata H, Hayano J, Miyake S, Kamada T, Kuno M, Kumashiro M. Assessment of autonomic function in traumatic quadriplegic and paraplegic patients by spectral analysis of heart rate variability. J Auton Nerv Syst 1995;54:225-34

[6] Grimm DR, De Meersman RE, Almenoff PL, Spungen AM, Bauman WA. Sympathovagal balance of the heart in subjects with spinal cord injury. Am J Physiol 1997;272:H835-842
[7] Bassingthwaite JB, Raymond GM. Evaluation of dispersional analysis method for fractal time series. Ann Biomed Eng 1995;23;491-505

[8] Pincus SM. Approximate entropy as a measure of system complexity. Proc Natl Acad Sci USA 1991;88:2297-301

[9] Peng CK, Havlin S, Stanley HE, Goldberger AL. Quantification of scaling exponents and crossover phenomena in nonstationary heartbeat time series. Chaos 1995;5:82-87

[10]Tulppo MP, Hughson RL, Makikallio TH, Juhani Airaksinen KE, Seppanen T, Huikuri HV. Effects of exercise and passive head-up tilt on fractal and complexity properties of heart rate dynamics. Am J Physiol 2001;280:H1081-7

Address for correspondence:

Giampiero Merati, MD.

Institute of Physical Exercise, Health and Sports (IEFSAS), Faculty of Exercise Science, University of Milan

Via Colombo 71, 20132 - Milan, Italy.

E-mail address: giampiero.merati@ unimi.it 